\title{
AVALIAÇÃO DO IMPACTO SOCIAL NO PROCESSO DE IMPLANTAÇÃO DA PRODUÇÃO INTEGRADA DE PÊSSEGOS NOS MUNICÍPIOS DE ARAUCÁRIA E LAPA - PARANÁ: um estudo de caso
}

Social impact assessment in the implementation of integrated production of peaches in A raucaria and L apa counties - Parana State (Brazil): a case study

\author{
Joel Ferreira Penteado Junior ${ }^{\mathrm{a}}$, Louise Larissa May De Mio ${ }^{\mathrm{b}}$, Geraldo Stachetti Rodrigues ${ }^{\mathrm{c}}$ \\ a Embrapa Florestas, Colombo, PR - Brasil, e-mail:joel@ cnpf.embrapa.br \\ b Universidade Federal do Paraná, (UFPR), Curitiba, PR - Brasil, e-mail maydemio@ ufpr.br \\ c Embrapa Labex E uropa, França, e-mail: stachetti-rodrigues@ agropolis.fr
}

\begin{abstract}
Resumo
Muitas inovações tecnológicas vêm sendo adotadas por produtores agrícolas para a implantação do sistema de produção integrada (PI) no Estado do Paraná. As tecnologias transferidas pela pesquisa redundam em impactos ligados à melhoria da qualidade de vida das pessoas vinculadas às atividades estudadas e são efetivas se há evidência concreta de que estão sendo cumpridos os objetivos sociais dos projetos. O presente trabalho avaliou por meio do aplicativo computacional Ambitec-Social, desenvolvido pela E mbrapa, os impactos sociais derivados da adoção da PI em dois estabelecimentos rurais, localizados nos municípios de Araucária e Lapa. Verificou-se que os índices de impacto, que podem variar de -15 a +15 no aplicativo empregado, foram de $+3,30$ para 0 estabelecimento de Araucária e de +2,77 para a Lapa. Os indicadores que mais se destacaram positivamente foram, 'Capacitação', 'Valor da propriedade', 'D edicação e Perfil do Responsável'.
\end{abstract}

Palavras-chave: Avaliação de impactos. Metodologia. Ambitec-Social.

\begin{abstract}
Many technological innovations have been adopted by farmers in the implementation of integrated production (IP) in Parana State. Knowing whether these innovations transferred by research effectively result in impacts related to enhancing the quality of life of people linked to the activities is necessary to verify that IP is meeting its social objectives. The present work evaluated the
\end{abstract}


social impacts resulting from the adoption of IP in two rural establishments located in Araucaria and Lapa counties, through the application of the Ambitec-Social assessment system, developed by Embrapa. Results show that impact indices, which may vary from -15 to +15 in the assessment system used, were +3.30 for the establishment in Araucaria and +2.77 , in Lapa. The indicators that stood out most positively were 'Training', 'Land value', 'Farmer capability and dedication'.

Keywords: Assessment of impacts. Methodology. Ambitec-Social.

\section{INTRODUÇÃO}

A Produção Integrada de Frutas (PIF) foi adotada no Brasil com o objetivo de produzir frutas de qualidade, priorizando a conservação de recursos naturais e a segurança do produtor e do consumidor (FACHINELLO et al., 2000). O s benefícios gerados a partir da utilização de sistemas de produção que privilegiam a conservação da biodiversidade, embora pouco estudados e de difícil quantificação, representam um importante benefício social, tanto no aspecto de conservação ambiental quanto de qualidade de vida. E é nesta dimensão que se encontra o principal aporte da PIF para a competitividade do agronegócio (EMBRAPA, 2007).

De acordo com Rodrigues (1998), Rodrigues et al. (2000), Rodrigues, Campanhola e Kitamura (2002), Rodrigues e Campanhola (2003), a dimensão social é parte indissociável das metodologias desenvolvidas para a avaliação dos impactos provocados pelas inovações tecnológicas aplicadas às atividades produtivas em estabelecimentos rurais. Assim, é importante identificar como e onde as tecnologias interferem no processo de produção e, a partir daí, saber como provocam modificações em diferentes aspectos da organização social (Q UIRINO , 1999), objetivando a mensuração da eficiência e 0 incremento do impacto social positivo (YEGANIANTZ; MACED 0, 2002).

A avaliação dos impactos sociais de tecnologias adotadas na realização das atividades produtivas rurais é a fase final da pesquisa e desenvolvimento, mas tão importante como as etapas de análise e de validação (TUPY et al., 2006). Conforme Yeganiantz e Macedo (2002), a geração de tecnologia deve demandar a inclusão dos conteúdos voltados para a qualidade de vida, e não simplesmente para os aspectos econômicos e técnico-produtivistas.

Uma ampla variedade de métodos de avaliação de impactos está disponível em trabalhos dedicados ao tema social e ambiental e inseridos em várias linhas metodológicas (RO D RIG UE S, 1998), sendo que cada método apresenta vantagens e desvantagens e trata mais adequadamente de problemas e objetivos específicos (CANTER, 1977). O presente estudo teve como objetivo utilizar o aplicativo A mbitec-Social para avaliar os impactos sociais promovidos pela implantação da PI de pêssego, em dois estabelecimentos agrícolas localizados em Araucária e Lapa, PR.

\section{MATERIAIS E MÉTODOS}

O estudo foi realizado nos anos de 2006 e 2007 nos municípios de Araucária e Lapa (PR) e trata da avaliação do impacto social provocado por projetos de PIF implantados pelo Grupo de Extensão, Ensino e Pesquisa, em Produção Integrada-G EEPPI, coordenados pela Universidade Federal do Paraná (UFPR) nos anos de 2002 a 2004. Em Araucária, a avaliação foi conduzida em um estabelecimento agrícola que dispõe de bom nível tecnológico e a área total da propriedade é de 217 hectares, onde a produção de pêssego e ameixa, apesar de utilizarem apenas 8\% da área, representa aproximadamente $50 \%$ do faturamento total do estabelecimento.

$\mathrm{Na}$ Lapa, o estabelecimento agrícola possui área total de 12 hectares, onde são cultivados pessegueiro, ameixeira e nectarineira. 
Avaliação do impacto social no processo de implantação da produção integrada de pêssegos nos municípios de Araucária e Lapa - Paraná

O Sistema de Avaliação de Impacto Social de Inovações Tecnológicas Agropecuárias (Ambitec-Social) empregado neste estudo consiste de um conjunto de 14 indicadores explicativos dos impactos sociais resultantes de uma dada atividade agropecuária no âmbito de um estabelecimento rural. Esses indicadores são agrupados em quatro aspectos de consideração, quais sejam: Emprego, Renda, Saúde e G estão e Administração.

A avaliação com o Sistema envolve duas etapas: a primeira refere-se ao processo de levantamento e coleta de dados gerais sobre a tecnologia (abrangência e influência) e a segunda etapa trata das avaliações de campo e entrevistas individuais com os responsáveis pela adoção da tecnologia.

Por causa da necessidade de compatibilizar a percepção adequada sobre os impactos avaliados nos diferentes indicadores, utilizou-se uma abordagem de interação, na qual os dados foram obtidos via vistoria de campo e entrevista com os responsáveis pelos estabelecimentos e com integrantes do GEPPI que implantaram e conhecem os resultados da adoção das tecnologias. Os coeficientes de alteração resultantes desses levantamentos só eram inseridos nas matrizes de ponderação componentes do Sistema quando se chegava a um consenso sobre os efeitos da PI em cada um dos indicadores avaliados.

A inserção desses coeficientes de alteração obtidos nos levantamentos de dados nas matrizes do sistema resulta na expressão automática dos índices de impacto social da tecnologia. Os índices de impacto são obtidos em procedimentos de ponderação por fatores relativos à escala da ocorrência da alteração e ao peso dos componentes do indicador.

O fator de ponderação para escala de ocorrência do componente explicita o espaço no qual ocorre a alteração no componente do indicador, conforme a situação específica de aplicação da tecnologia, e pode ser: 1) Pontual, quando o efeito da tecnologia no componente restringe-se ao campo de cultivo ou unidade produtiva na qual esteja ocorrendo a alteração (fator de ponderação 1); 2) Local, quando 0 efeito se faz sentir externamente a essa unidade produtiva, porém confinado aos limites da propriedade (fator de ponderação 2); 3) No entorno, quando o efeito abrange além dos limites da propriedade (fator de ponderação 5). 0 fator de ponderação da escala da ocorrência implica a multiplicação do coeficiente de alteração do componente por um valor predeterminado.

O segundo fator de ponderação incluído nas matrizes de avaliação de impacto da inovação tecnológica é a importância do componente para a formação do indicador de impacto ambiental. 0 sistema de pontuação utilizado reflete a avaliação sensorial sobre a alteração do indicador, a alteração refere-se ao desempenho da atividade desenvolvida com a inovação tecnológica, em comparação à atividade desenvolvida antes da adoção. Para um grande aumento no componente o valor da pontuação é (+3); para um moderado aumento no componente é (+1); quando componente inalterado é (0); para uma moderada diminuição é (-1) e quando ocorre grande diminuição é (-3).

São atribuídos, ainda, pesos para os componentes, que variam conforme a sua quantidade na formação do indicador, sendo, portanto, usados para normalizar os indicadores conforme 0 teste de sensibilidade aplicado caso a caso (GIRARDIN et al., 1999). Os pesos dos componentes expressos nas matrizes podem ser alterados pelo usuário do sistema para melhor refletir situações específicas de avaliação, nas quais se pretenda enfatizar alguns dos componentes, desde que o peso total dos componentes para um dado indicador seja igual à unidade (1). Cabe ressaltar que para uma mesma tecnologia, mesmo quando utilizada em diferentes sistemas naturais, os valores dos pesos dos componentes dos indicadores devem permanecer os mesmos, a fim de que os resultados sejam comparáveis.

O s resultados finais da avaliação de impacto são expressos graficamente, com os indicadores considerados em seu conjunto para composição do Índice de Impacto Social da Inovação Tecnológica. A escala padronizada no sistema varia entre -15 e +15 , normalizada para todos os indicadores individualmente e para o Índice G eral de Impacto Social da Tecnologia. É importante esclarecer que 0 método traz como norma de avaliação a adequação tecnológica definida como minimização de impactos negativos, em qualquer dos indicadores. Para maiores detalhes sobre o Sistema Ambitec-Social ver Rodrigues, Campanhola e Kitamura (2002), Rodrigues et al. (2005, 2006). ${ }^{1}$

\footnotetext{
${ }^{1}$ Publicações referentes ao sistemaA mbitec-Agro e seus módulos, bem como as planilhas para aplicação em campo podem ser obtidas pelo acesso à página da Embrapa Meio Ambiente na internet. D isponível em: < http:/ / www.cnpma.embrapa.br/ forms/ index.php3?func=softwma $>$.
} 


\section{RESULTADOS}

Não encontramos na literatura nenhum trabalho referente à avaliação de impacto social da adoção de Produção Integrada de pêssegos, tampouco a utilização do aplicativo Ambitec-Social para avaliação de estabelecimentos agrícolas que cultivam pêssego no sistema de produção integrada. Assim, os resultados obtidos neste estudo (Figura 1), são comparados com trabalhos desenvolvidos com outras culturas.

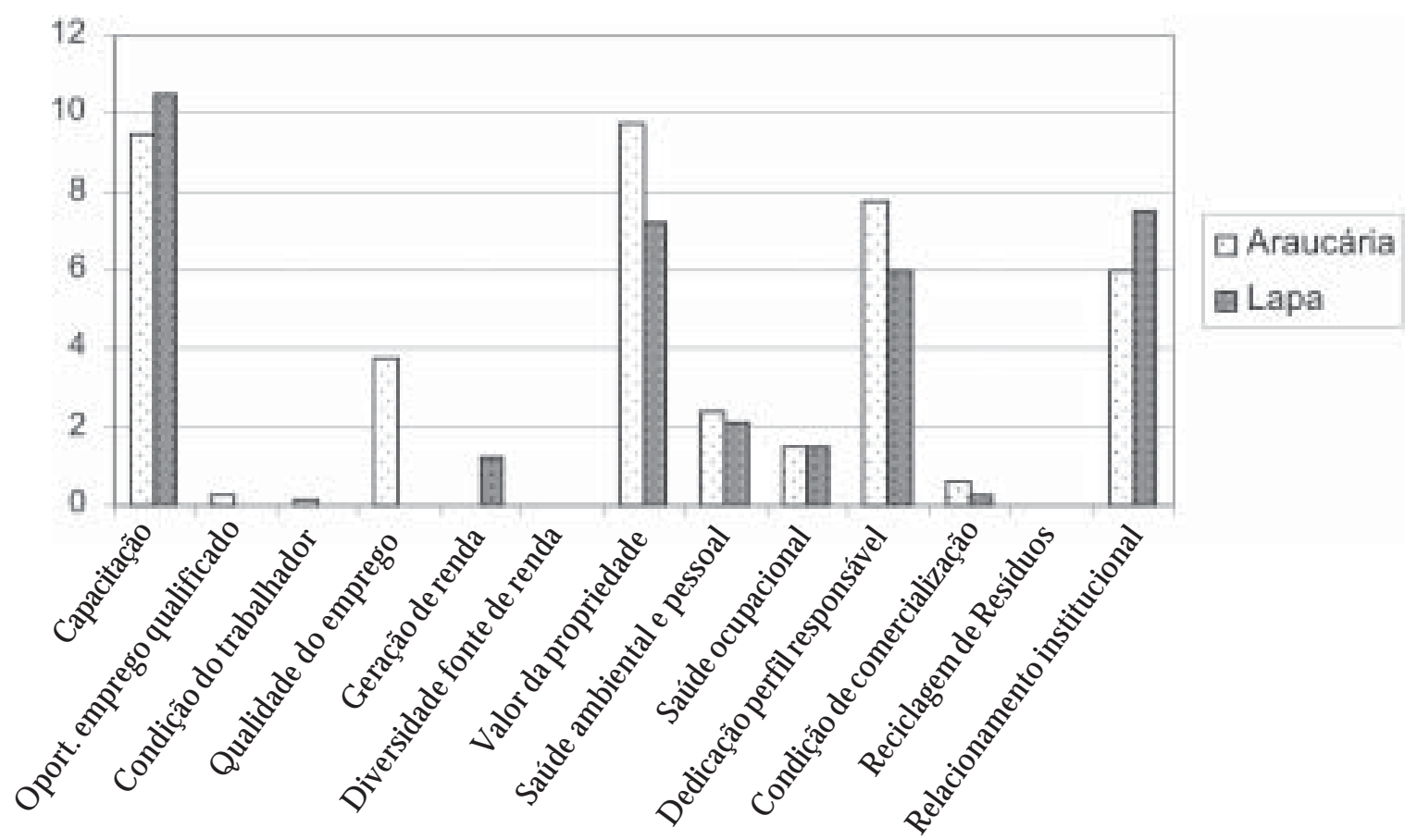

FIGURA 1 - Avaliação final, apresentando a ponderação dos coeficientes e indicadores de Impacto Social com o sistema Ambitec para os estabelecimentos agrícolas de produção integrada de pêssego em Araucária e Lapa, PR

O indicador Capacitação considera três tipos de treinamentos, quais sejam: treinamento local de curta duração, especialização de curta duração, e cursos oficiais regulares de ensino. A PI propiciou grande aumento na realização de treinamentos e especialização em níveis básicos e técnicos, resultando em importante impacto positivo no estabelecimento agrícola de Araucária. Isto se deve ao fato de todos os trabalhadores terem participado de treinamentos e cursos diretamente relacionados às novas práticas preconizadas pela PI. No estabelecimento de Lapa, a PI propiciou a necessidade e o grande aumento de treinamentos e especialização em níveis básicos e técnicos, resultando em impacto positivo. Mais de 50\% dos trabalhadores participaram de treinamentos e cursos diretamente relacionados à PI. Estas capacitações foram contratadas pelos proprietários dos estabelecimentos e oferecidas pela EMATER-PR e UFPR. Jacometi (2007), em avaliação social do impacto de certificação de limão tahiti no Estado de São Paulo, demonstrou a importância da PI e da certificação na melhoria da qualidade da mão-de-obra agrícola, o que se confirma no presente trabalho.

$\mathrm{O}$ indicador 0 portunidade de emprego considera a qualificação e a origem do trabalhador ocupado, seja o trabalhador proveniente da região, do local ou município, ou da própria propriedade. A ponderação foi realizada sobre a porcentagem do pessoal ocupado na atividade à qual se aplicava a PI. Já a qualificação exigida para o emprego proporcionado pela PI foi avaliada como braçal, braçal especializado, técnico médio, e técnico de nível superior. A introdução da PI propiciou ao menos um pequeno aumento na oportunidade de novos empregos, para trabalhadores qualificados, do local e que foram treinados na propriedade em Araucária. No estabelecimento rural da Lapa, nenhum efeito ocorreu em relação ao engajamento de trabalhadores, resultado semelhante ao encontrado por Jacometi (2007) em avaliação da certificação de produção de limão, e Tupy et al. (2006) nas avaliações das tecnologias da Embrapa Pecuária Sudeste (Tabelas 1 e 2). 
Avaliação do impacto social no processo de implantação da produção integrada de pêssegos nos municípios de Araucária e Lapa - Paraná

TABELA 1 - Matriz dos indicadores e componentes do Impacto Social do sistema Ambitec-Social, para 0 estabelecimento agrícola de Araucária, PR

\begin{tabular}{|c|c|c|c|c|}
\hline $\begin{array}{c}\text { Indicadores e componentes } \\
\text { para avaliaçãoAmbiental - } \\
\text { Araucária, PR }\end{array}$ & $\begin{array}{c}\text { Fator de } \\
\text { ponderação } \\
\text { da escala de } \\
\text { ocorrência (a) }\end{array}$ & $\begin{array}{c}\text { Fator de } \\
\text { ponderação } \\
\text { peso do } \\
\text { componente (b) }\end{array}$ & $\begin{array}{l}\text { Índice de } \\
\text { Impacto (c) }\end{array}$ & $\begin{array}{l}\text { Coeficiente } \\
\text { de impacto } \\
\left(\mathbf{a}^{*} \mathbf{b}^{*} \mathbf{c}\right)\end{array}$ \\
\hline \multicolumn{5}{|l|}{ Indicador- Capacitação } \\
\hline Componentelocal de curta duração & 1 & 0,25 & 3 & 0,75 \\
\hline Componente longa duracão & 1 & 0,25 & 3 & 0,75 \\
\hline Componentebásico & 1 & 0,1 & 3 & 0,3 \\
\hline Componente médio & 1 & 0,1 & 1 & 0,1 \\
\hline Coeficiente de impacto & & & & 1,9 \\
\hline \multicolumn{5}{|l|}{$\begin{array}{l}\text { Indicador-Oportunidade } \\
\text { de emprego local }\end{array}$} \\
\hline Componente origem local & 1 & 0,2 & 1 & 0,2 \\
\hline $\begin{array}{l}\text { Componente qualificação } \\
\text { braçal especializado }\end{array}$ & 1 & 0,05 & 1 & 0,05 \\
\hline Coeficiente de impacto & & & & 0,25 \\
\hline \multicolumn{5}{|l|}{$\begin{array}{l}\text { Indicador - O ferta de } \\
\text { emprego e condição } \\
\text { do trabalhador }\end{array}$} \\
\hline Componente permanente & 1 & 0,15 & 1 & 0,05 \\
\hline Coeficiente de impacto & & & & 0,15 \\
\hline \multicolumn{5}{|l|}{$\begin{array}{l}\text { Indicador-Q ualidade } \\
\text { do emprego }\end{array}$} \\
\hline Componente registro & 1 & 0,15 & 3 & 0,45 \\
\hline $\begin{array}{l}\text { Componente contribuição } \\
\text { previdenciária }\end{array}$ & 1 & 0,10 & 3 & 0,3 \\
\hline Coeficiente de impacto & & & & 0,75 \\
\hline \multicolumn{5}{|l|}{$\begin{array}{l}\text { Indicador - Valor } \\
\text { da propriedade }\end{array}$} \\
\hline $\begin{array}{l}\text { Componente investimento } \\
\text { em benfeitorias }\end{array}$ & 1 & 0,25 & 3 & 0,75 \\
\hline $\begin{array}{l}\text { Componente conservação } \\
\text { recursosnaturais }\end{array}$ & 1 & 0,25 & 3 & 0,75 \\
\hline $\begin{array}{l}\text { Componente conformidade } \\
\text { legislação }\end{array}$ & 1 & 0,15 & 3 & 0,45 \\
\hline Coeficiente de impacto & & & & 1,95 \\
\hline \multicolumn{5}{|l|}{ Indicador - Saúde ambiental } \\
\hline $\begin{array}{l}\text { Componente emissão de } \\
\text { poluentes hídricos }\end{array}$ & 2 & 0,2 & -3 & 1,2 \\
\hline $\begin{array}{l}\text { Componente geração de } \\
\text { contaminantes do solo }\end{array}$ & 1 & 0,2 & -3 & 0,6 \\
\hline Coeficiente de impacto & & & & 1,8 \\
\hline
\end{tabular}


Indicador-Segurança e

Saúde 0 cupacional

Componente: periculosidade

Componente: agentes químicos

$1 \quad 0,25$

0,25

3

0,75

Coeficiente deimpacto

Indicador- Dedicaçãoe

perfil do responsável

Componente: capacitação

dirigida à atividade

Componente: horas de dedicação

10,25

3

0,75

Componente: uso de sistema

contábil

Componente: aplicação de modelo formal de planejamento

Componente: sistema

decertificação

Coeficiente deimpacto

1,55

\section{Indicador-Condição de}

comencialização

Componente: processamento

Componente: armazenamento

1

0,15

0,15

3

0,45

1

0,2

0,6

1

0,15

1

0,2

1

0,15

1

0,15

Coeficiente deimpacto

0,9

\section{Indicador- Reciclagem}

deresíduos

Indicador- Relacionamento

institucional

Componente: Assistência técnica

Componente: Utilização

assessorialegal

Componente: Capacitação

contínua empregados

10,2

$1 \quad 0,15$

1

Coeficiente deimpacto
0,15

3

3

0,6

0,15

1

0,15

1,2 
Avaliação do impacto social no processo de implantação da produção integrada de pêssegos nos municípios de Araucária e Lapa - Paraná

TABELA 2 - Matriz dos indicadores e componentes do impacto social do sistema Ambitec-Social, para 0 estabelecimento agrícola, para Lapa, PR

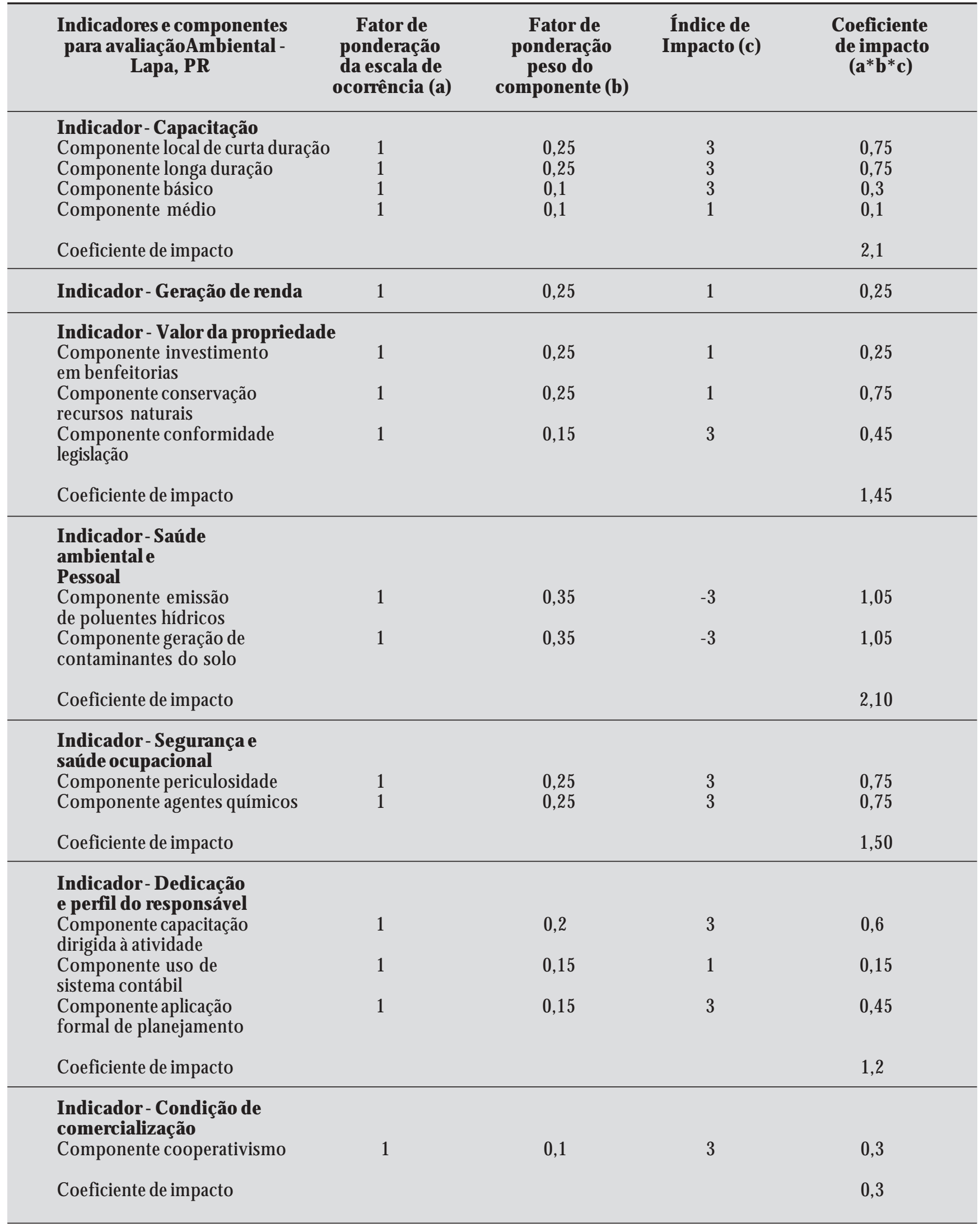


Indicador - Reciclagem de

resíduos

\section{Indicador- Relacionamento}

institucional

Componente assistência técnica

Componente asso ciativismo

Componente utilização

assessorialegal

Componente capacitação contínua

empregados

Coeficiente de impacto
0

$\begin{array}{llll}1 & 0,2 & 3 & 0,6 \\ 1 & 0,2 & 3 & 0,6 \\ 1 & 0,15 & 1 & 0,15 \\ 1 & 0,15 & 1 & 0,15\end{array}$

1,5

No indicador 0 ferta de emprego e condicão do trabalhador foram abordadas alterações na oferta quantitativa de emprego por força da adoção da implantação da PI. Consideraram-se os regimes de trabalho temporário, permanente, parceiros/ meeiros ou familiares. A PI propiciou a geração de novos empregos permanentes no estabelecimento rural de A raucária, onde esses novos contratados se dedicam exclusivamente à PI. Assim, o impacto foi positivo nesse indicador. No estabelecimento da Lapa, a PI não propiciou a geração de novos empregos.

O indicador Q ualidade do emprego se referiu a todos os trabalhadores do estabelecimento, engajados em consequência da adoção da PI. O emprego foi qualificado segundo os principais parâmetros legais de atendimento a condições básicas, como idade mínima, jornada máxima de trabalho, formalidade e auxílios e benefícios previstos pelas leis trabalhistas brasileiras. Em Araucária, a avaliação desse indicador apontou efeito positivo, por causa do aumento de empregados registrados. Na Lapa, a adoção da PI não causou impactos em nenhum dos componentes, resultado similar ao verificado nos trabalhos citados na literatura.

Quanto à $\mathrm{G}$ eração de renda, o indicador é condicionado pela tendência dos atributos de segurança, estabilidade, distribuição e montante. A introdução da PI praticamente não trouxe melhorias nos componentes de renda em nenhum dos dois estabelecimentos rurais estudados. Apesar da PI proporcionar a redução de aplicação de agrotóxicos e melhoria da qualidade do produto, os preços são os mesmos que os dos produzidos convencionalmente e sujeitos às mesmas condições de mercado anteriores à adoção das tecnologias.

$\mathrm{O}$ indicador $\mathrm{V}$ alor da propriedade refere-se à alteração no valor da terra sob efeito da adoção da PI. As causas podem ser representadas por investimento em benfeitorias, qualidade e conservação dos recursos naturais, conformidade com a legislação e melhorias em infraestrutura. Nas duas propriedades estudadas, esse indicador foi um dos que apresentou maior impacto. 0 investimento em benfeitorias como barracões, abastecedouro, captação da água usada para lavagem de implementos, instalação específica para armazenamento e separação de agrotóxicos, entre outros, proporcionaram impactos positivos nos dois estabelecimentos rurais estudados.

O indicador Saúde ambiental e pessoal considerou alterações advindas da adoção da PI na existência de focos de doenças endêmicas, emissões de poluentes sejam atmosféricos, hídricos ou para o solo, e dificuldade de acesso a esporte e lazer. Em ambos os estabelecimentos, o preparo das caldas de agroquímicos, que ocorria próximo a fontes de água, após a adoção da PI se executa em locais apropriados, construídos especialmente para este fim, implicando assim em um impacto positivo.

Q uanto àS egurança eSaúde ocupacional foi retratadaa exposição de trabalhadores à periculosidade e fatores de insalubridade devido à adoção da PI. Nos dois estabelecimentos rurais estudados a PI propiciou a diminuição da exposição a efeitos potencialmente perigosos e a exclusão e diminuição da exposição a agentes químicos. Nas duas situações, após a implantação da PI, assegurou-se a todos os empregados o uso de equipamentos de proteção individual (EPIs) adequados para a preparação e aplicação de agroquímicos, propiciando uma diminuição na exposição dos trabalhadores, o que causou nos estabelecimentos impacto positivo. 
O indicador D edicação e perfil do responsável, é constituído por variáveis necessárias para 0 gerenciamento, tais como capacitação dirigida à atividade, horas de dedicação, engajamento familiar nos negócios do estabelecimento, uso de sistema contábil, aplicação de modelo formal de planejamento e sistema de certificação. A PI exigiu aprimoramento na capacitação e registro das atividades com 0 preenchimento da caderneta de campo e constante planejamento das atividades. No estabelecimento rural de Araucária o impacto foi positivo. Na Lapa, além de registro formal das atividades, o engajamento da família e do produtor responsável pelo estabelecimento gerou um importante impacto positivo neste indicador, provocado pela adoção da PI.

O indicador Condicão de comercializacạa inclui os atributos descritores da inclusão no mercado dos produtos obtidos pela atividade à qual se aplica a tecnologia em avaliação. Na propriedade de Araucária a PI resultou em moderada melhoria do sistema de comercialização, pela alteração nos componentes de armazenamento e processamento no local, proporcionando impacto social positivo neste indicador. No estabelecimento agrícola da Lapa, a PI não resultou em melhoria no sistema de comercialização. No entanto, serviu como um impulso para o engajamento do produtor no sistema de cooperativa, o qual pode proporcionar condições para melhorias futuras para o sistema, como apontado no indicador que segue.

0 indicador Relacionamento institucional abordou atributos de acesso à assistência técnica, associativismo, assessoria legal e vistoria. Foi registrada uma importante alteração positiva nos componentes relacionados à assistência técnica em Araucária, as quais ocorreram devido à necessidade de cumprir de maneira adequada as exigências da adoção da PI. No estabelecimento da Lapa houve também uma alteração positiva nos componentes relacionados à assistência técnica, as quais ocorreram por causa da variedade de tecnologias adotadas pela PI que necessitavam de constante assessoramento. Um impacto positivo também ocorreu no componente associativismo/ cooperativismo, dada a aproximação do responsável pelo estabelecimento com uma cooperativa de produtores.

Na Figura 1 são apresentados os resultados dos índices de impacto social da implantação da PI de pêssegos nos estabelecimentos rurais estudados nos municípios de Araucária e Lapa. 0 índice geral de impacto social da PI obtido no estabelecimento de Araucária (Figura 2) alcançou valor positivo igual a 3,30 de um valor máximo possível de 15. Dentre todos os indicadores, nenhum resultou em impacto negativo e entre os que apresentaram maior alteração se destacaram, em ordem decrescente, a capacitação, o valor da propriedade, a dedicação do perfil do proprietário, o relacionamento institucional, a qualidade do emprego e a saúde ocupacional.

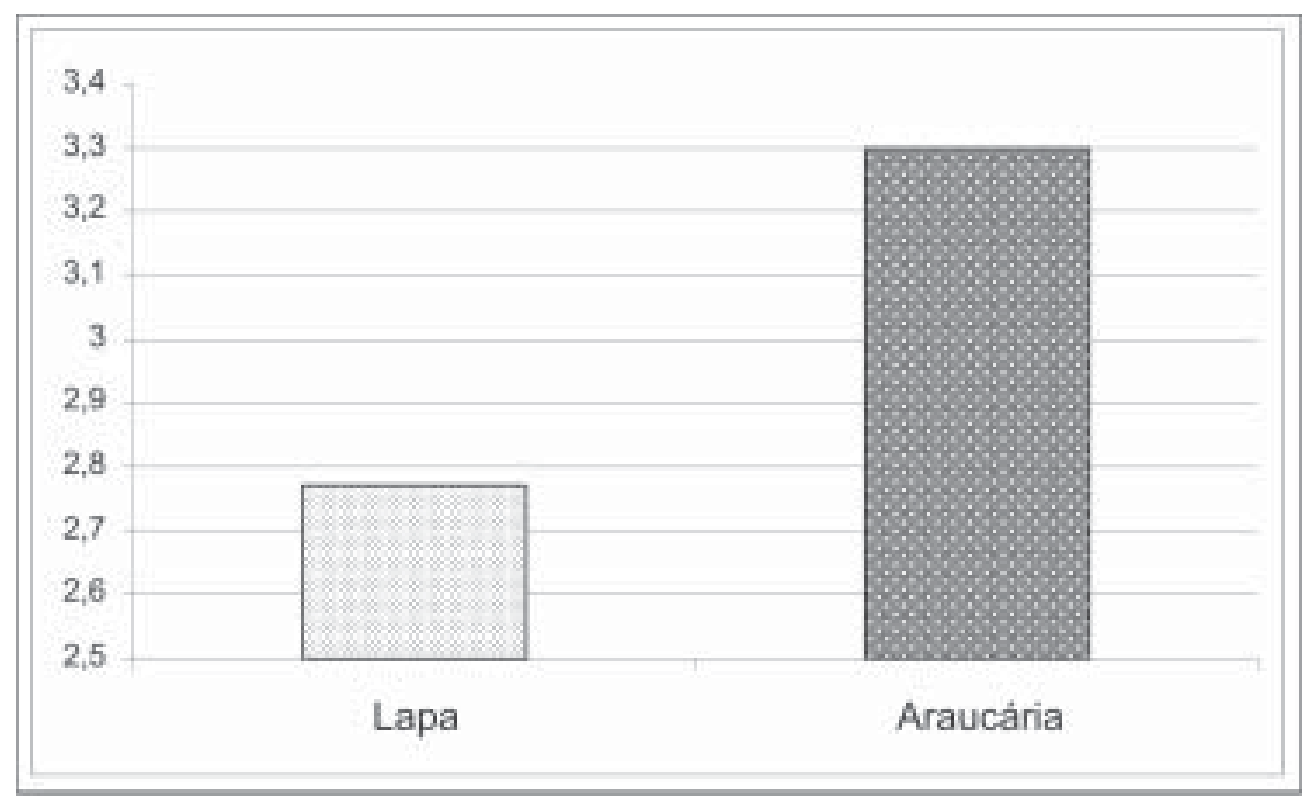

FIGURA 2 - Avaliação final, apresentando o índice de impacto final de Impacto Social com o sistema A mbitec para os pomares de produção integrada de pêssego em Araucária e Lapa, PR 
No estabelecimento da Lapa, conforme apresentado na Figura 2, o índice geral de impacto social da PI de Pêssego alcançou o valor de 2,77, de uma escala que pode variar de -15 a +15 . D entre todos os indicadores, os que apresentaram maiores benefícios foram, em ordem decrescente, a capacitação, 0 valor da propriedade, a dedicação do perfil do proprietário e o relacionamento institucional. Nenhum resultou em impacto negativo, o que indica que a inovação tecnológica como um todo apresenta contribuição social positiva, no âmbito dos estabelecimentos estudados, e qualifica-se para indicação de adoção por outros produtores dedicados à produção de pêssegos.

Os valores obtidos neste trabalho são equivalentes aos encontrados por Jacometi (2007) em avaliação do impacto social provenientes da certificação EUREPGAP em 14 estabelecimentos agrícolas produtores de limão tahiti no Estado de São Paulo. Os índices obtidos naquela avaliação, empregando a mesma base metodológica, variaram de 2,14 a 3,54, sendo a dedicação e perfil do responsável e a reciclagem de resíduos os indicadores que mais se destacaram.

Ávila (2006), em apresentação de resultados de avaliação de impactos de tecnologias geradas pela Embrapa, informou índice geral de impactos de 0,51 para manejo de pragas de feijoeiro comum; 0,65 para cultivar de cebola Alfa para plantio de verão; 2,00 para cultivar de algodão com fibra colorida; 1,18 para cultivar de mamona para o Nordeste brasileiro. A implantação da PI, estudada no presente trabalho, resultou em índices positivos de impacto e, com o decorrer do tempo, a repetição das práticas adotadas pelos produtores poderá proporcionar melhoria das condições e desempenho dos indicadores sociais. Assim, avaliações neste sentido devem ser constantes.

\section{CONCLUSÕES}

Em ambos os estabelecimentos o impacto foi positivo em todos os aspectos focados na análise, atendendo à norma estabelecida pelo método, de buscar desenvolvimento tecnológico evitandose impactos sociais negativos.

Os destaques de impactos positivos, nos dois estabelecimentos, foram: Capacitação, Valor da propriedade, D edicação e perfil do responsável, Q ualidade do emprego e Relacionamento institucional.

\section{REFERÊNCIAS}

ÁVILA, A. F. D. Experiência da Embrapa em avaliação de impactos da pesquisa agropecuária.

São José dos Campos. 2006. Disponível em: < http:/ / www.inpe.br/ dspace/ bitstream/ 123456789/ 261/1/Impactos_Embrapa_Fabio-Avila_G T08.pdf>. Acesso em: 20 jul. 2007.

CANTER, L. W. Environmental impact assessment. New York: McG raw-Hill, 1977.

EMPRESA BRA SILEIRA DE PESQUISA AGROPECUÁRIA - EMBRAPA. Sistema de produção

de maçã. Versão eletrônica. D isponível em: < http:/ / sistemasdeproducao.cnptia.embrapa.br/ FontesHTML/ Maca/ ProducaoIntegradaMaca/ index.htm>. Acesso em: 15 out. 2007.

FACHINELLO, J. C. et al. Avaliação do sistema de produção integrada de pêssego de conserva na região de Pelotas-safra 1999-2000. In: SEMINÁRIO SOBRE PRODUÇÃO INTEGRADA DE FRUTAS, 2., 2000, Bento Gonçalves. Anais... Bento Gonçalves: Embrapa Uva e Vinho, 2000. p. 78-85.

GIRARD IN, P.; BOCK STALLER, C.; WERF, H. van der. Indicators: tools to evaluate the environmental impacts of farming systems. J. Sustain. Agric., Binghamton, v. 13, n. 4, p. 5-21, 1999.

HOFFMANN, C.; BUSKE, E. O. Cooperativismo uma alternativa para pequenos fruticultores. In: SEMINÁRIO DE PRO DUÇÃO INTEGRADA: COMO EVOLUIR E CONCRETIZAR: CURSO DE PRODUÇÃO INTEG RADA, 1., 2006. Anais... Universidade Federal do Paraná, Curitiba, 2006. p. 24-31. 
INSTITUTO PARANAENSE DE ASSISTÊNCIA TÉCNICA E EXTENSÃO RURAL EMATER. Relatório de atividades da EMATER - Paraná. Curitiba: EMATER, 2004.

JACOMETI, W. A. Certificação eurepgap na fruticultura: avaliação de impactos ambientais e sociais. 2007. 135 f. D issertação (Mestrado em D esenvolvimento Regional e Meio Ambiente) Centro Universitário de Araraquara, Araraquara, 2007.

MACED O, M. M. C.; YEG ANIANTZ, L. Avaliação de impacto social de pesquisa agropecuária: a busca de uma metodologia baseada em indicadores. Brasília: Embrapa/ SEA, 2000.

QUIRINO, T. R. et al. Impacto agroambiental. São Paulo: Edgard Blücher, 1999.

RODRIGUES, G. S. Avaliação de impactos ambientais em projetos de pesquisas: fundamentos, princípios e introdução à metodologia. Jaguariúna: Embrapa Meio Ambiente, 1998. (D ocumentos, 14).

ROD RIGUES, G. S.; CAMPANHO LA, C. Sistema integrado de avaliação de impacto ambiental aplicado a atividades do novo rural. Pesq. Agropec. Bras., Brasília, v. 38, n. 4, p. 445-451, 2003.

ROD RIGUES, G. S.; CAMPANHOLA, C.; KITAMURA, P. C. Avaliação de impacto ambiental da inovação tecnológica agropecuária: um sistema de avaliação para o contexto institucional de P\&D. Cad. Cien.Tecnol., Brasília, v. 19, n. 3, p. 349-375, 2002.

RO D RIG UES, G. S. et al. Avaliação de impactos ambientais em projetos de pesquisa II: avaliação da formulação de projetos. Jaguariúna: Embrapa Meio Ambiente, 2000. (D ocumentos, 10).

. Sistema de avaliação de impacto social da inovação tecnológica agropecuária (Ā'mbiètc-Social). Jaguariúna: Embrapa Meio Ambiente, 2005.

. Avaliação sócio-ambiental da integração tecnológica Embrapa Pecuária Sudeste para produção leiteira na agricultura familiar. Agricultura em São Paulo, São Paulo, v. 53, n. 2, p. 35-48, 2006.

TUPY, O. et al. Avaliação dos impactos econômicos, sociais e ambientais de tecnologias da Embrapa Pecuária Sudeste. São Carlos: Embrapa Pecuária Sudeste, 2006. (D ocumentos, 08).

YEGANIANTZ, L.; MACEDO, M. M. C. Avaliação de impacto social de pesquisa agropecuária: a busca de uma metodologia baseada em indicadores. Brasília: Embrapa Informação Tecnológica, 2002. (Texto para Discussão; 13).

Recebido: 15/ 06/ 2008

Received: 06/ 15/ 2008

Aprovado: 22/01/ 2009

A pproved: 01/22/ 2009

Revisado: $17 / 08 / 2009$

Reviewed: 08/ 17/ 2009 